IOANNIS K. Argyros (Lawton, OK)

SAÏD Hilout (Poitiers)

\title{
CONVERGENCE DOMAINS UNDER ZABREJKO-ZINČENKO CONDITIONS USING RECURRENT FUNCTIONS
}

Abstract. We provide a semilocal convergence analysis for Newton-type methods using our idea of recurrent functions in a Banach space setting. We use Zabrejko-Zinčenko conditions. In particular, we show that the convergence domains given before can be extended under the same computational cost. Numerical examples are also provided to show that we can solve equations in cases not covered before.

1. Introduction. In this study we are concerned with the problem of approximating a locally unique solution $x^{\star}$ of the equation

$$
F(x)+G(x)=0,
$$

where $F$ is a Fréchet-differentiable operator defined on an open convex subset $\mathcal{D}$ of a Banach space $\mathcal{X}$ with values in a Banach space $\mathcal{Y}$, and $G: \mathcal{D} \rightarrow \mathcal{Y}$ is a continuous operator.

A large number of problems in applied mathematics and also in engineering are solved by finding solutions of equations in the form (1.1) [4].

We shall use the Newton-type method (NTM)

$$
\begin{aligned}
& y_{n+1}=y_{n}-A\left(y_{n}\right)^{-1} P\left(y_{n}\right) \quad(n \geq 0) \quad\left(y_{0} \in \mathcal{D}\right), \\
& P(x)=F(x)+G(x) \quad(x \in \mathcal{D}),
\end{aligned}
$$

where $A \in \mathcal{L}(\mathcal{X}, \mathcal{Y})$, to generate a sequence approximating $x^{\star}$.

2010 Mathematics Subject Classification: 65G99, 65H10, 65B05, 65N30, 47H17, $49 \mathrm{M} 15$. Key words and phrases: Newton-type methods, recurrent functions, Banach space, Zabrejko-Zinčenko-type conditions, Kantorovich hypothesis, majorizing sequences, Green's kernel. 
(a) If

(b) If

$$
A(x)=F^{\prime}(x) \quad(x \in \mathcal{D}),
$$

we obtain the Zabrejko-Nguen iteration [26]:

$$
y_{n+1}=y_{n}-F^{\prime}\left(y_{n}\right)^{-1} P\left(y_{n}\right) \quad(n \geq 0), \quad\left(y_{0} \in \mathcal{D}\right) .
$$

$$
A(x)=F^{\prime}(x)+[x, y ; G] \quad(x \in \mathcal{D}),
$$

where $[x, y ; F]$ is a divided difference of order one for the operator $G$, then we obtain an iteration faster than $(1.4)$, first considered by Cătinaş [9]:

$$
y_{n+1}=y_{n}-\left(F^{\prime}\left(y_{n}\right)+\left[y_{n}, y_{n-1} ; G\right]\right)^{-1} P\left(y_{n}\right) \quad(n \geq 0) \quad\left(y_{-1}, y_{0} \in \mathcal{D}\right) \text {. }
$$

(c) If

$$
A(x)=F^{\prime}(x), \quad G(x)=0 \quad(x \in \mathcal{D}),
$$

then (NTM) reduces to Newton's method:

$$
x_{n+1}=x_{n}-F^{\prime}\left(x_{n}\right)^{-1} F\left(x_{n}\right) \quad(n \geq 0), \quad\left(x_{0} \in \mathcal{D}\right) .
$$

Several other choices are possible [4]-[7].

A local as well as a semilocal convergence analysis for all these methods has been provided by many authors under Lipschitz-type conditions [1]-[3], [11]-[27].

A survey of such results can be found in [4], and the references there. We also refer the reader to the elegant related works by Proinov [22], 23], and Ezquerro-Hernández [15] whose results are also improved here in at least the Newton's method case (see Section 3).

Let $x^{0} \in \mathcal{D}$ and $R>0$ be such that

$$
U\left(x^{0}, R\right)=\left\{x \in \mathcal{X}:\left\|x-x^{0}\right\|<R\right\} \subseteq \mathcal{D} .
$$

Chen and Yamamoto [1] provided a semilocal convergence for (NTM) for $y^{0} \in U\left(x^{0}, R\right)$ under the condition

$(\mathcal{C}) \quad A\left(x^{0}\right)$ exists, and for any $x, y \in \bar{U}\left(x^{0}, R\right)$ :

$$
\begin{aligned}
& \left\|A\left(x^{0}\right)^{-1}\left(A(x)-A\left(x^{0}\right)\right)\right\| \leq \overline{\nu_{0}}\left(\left\|x-x^{0}\right\|\right)+a, \\
& \begin{array}{l}
\left\|A\left(x^{0}\right)^{-1}\left(F^{\prime}(x+t(x-y))-A(x)\right)\right\| \\
\leq \bar{\nu}\left(\left\|x-x^{0}\right\|+t\|y-x\|\right)-\overline{\nu_{0}}\left(\left\|x-x^{0}\right\|\right)+b, \quad t \in[0,1], \\
\left\|A\left(x^{0}\right)^{-1}(G(x)-G(y))\right\| \leq \omega(r)\|x-y\|,
\end{array}
\end{aligned}
$$

where $\bar{\nu}(r+t)-\overline{\nu_{0}}(r), t \geq 0$, and $\omega(r)$ are non-decreasing, non-negative functions with

$$
\omega(0)=\overline{\nu_{0}}(0)=\bar{\nu}(0)=0,
$$


$\overline{\nu_{0}}(r)$ is differentiable, ${\overline{\nu_{0}}}^{\prime}(r)>0$ at every point of $[0, R]$, and the constants $a, b$ satisfy $a, b \geq 0$, and $a+b<1$.

Set

$$
\begin{gathered}
\left\|A\left(x^{0}\right)^{-1} P\left(x^{0}\right)\right\| \leq \eta, \\
\phi(r)=\eta-r+\int_{0}^{1} \bar{\nu}(t) d t, \quad \psi(r)=e \int_{0}^{r} \omega(t) d t, \\
\chi(r)=\phi(r)+\psi(r)+(a+b) r .
\end{gathered}
$$

Further, assume

$$
\chi(R) \leq 0,
$$

and define a scalar sequence $\left\{s_{n}\right\}$ by

$$
s_{0} \in[0, R], \quad s_{n+1}=s_{n}+\frac{u\left(s_{n}\right)}{p\left(s_{n}\right)} \quad(n \geq 0),
$$

where

$$
u(r)=\chi(r)-\chi^{\star}, \quad p(r)=1-\overline{\nu_{0}}(r)-a,
$$

$\chi^{\star}$ is the minimal value of $\chi(r)$ in $[0, R]$, and $s^{\star}$ denotes the minimal point. Moreover, $t^{\star}$ denotes the unique zero of $\chi$ in $\left(0, s^{\star}\right]$.

Under these assumptions, there exists a unique solution $x^{\star} \in \bar{U}\left(x^{0}, t^{\star}\right)$ such that

$$
\left\|y_{n+1}-y_{n}\right\| \leq s_{n+1}-s_{n}, \quad\left\|x^{\star}-y_{n}\right\| \leq s^{\star}-s_{n} .
$$

We shall use the more general set of conditions $(\mathcal{H}) \equiv\left(\mathcal{H}_{1}\right)-\left(\mathcal{H}_{4}\right)$, where $\left(\mathcal{H}_{1}\right) \quad A\left(x^{0}\right)$ exists, and for any $x, y \in \bar{U}\left(x^{0}, R\right)(0<r \leq R)$,

$$
\left\|A\left(x^{0}\right)^{-1}\left(A(x)-A\left(x^{0}\right)\right)\right\| \leq \nu_{0}\left(\left\|x-x^{0}\right\|\right)+a,
$$

$\left(\mathcal{H}_{2}\right) \quad\left\|A\left(x^{0}\right)^{-1}\left(F^{\prime}(x+t(x-y))-A(x)\right)\right\|$

$$
\leq \nu(t\|y-x\|)+\omega_{0}\left(\left\|x-x^{0}\right\|\right)+b, \quad t \in[0,1],
$$

$\left(\mathcal{H}_{3}\right) \quad\left\|A\left(x^{0}\right)^{-1}(G(x)-G(y))\right\| \leq \omega(r)\|x-y\|$,

$\left(\mathcal{H}_{4}\right) \quad \bar{U}\left(x^{0}, R\right) \subseteq \mathcal{D}$,

where $\nu_{0}, \nu$, and $\omega_{0}$ are non-decreasing, non-negative functions on $[0, R]$ with

$$
\nu_{0}(0)=\nu(0)=\omega_{0}(0)=\omega(0)=0,
$$

and $a$ and $b$ are non-negative constants. Some more hypotheses are given in Lemma 2.2.

A semilocal convergence analysis is provided in Section 2 of this study under the $(\mathcal{H})$ conditions, whereas in Section 3 we compare the two sets of hypotheses. Numerical examples are also provided in Section 3 to show how we can solve equations in cases not covered before [1]-[27. 
2. Semilocal convergence analysis of (NTM). We need to define some parameters, functions and sequences.

Definition 2.1. Let $y_{0} \in U\left(x^{0}, r\right)$. Define parameters $r_{0}, r_{1}$, iteration $\left\{r_{n}\right\}$, functions $f_{n}, h_{n}, p_{n}$ on $[0,1)$, and $q$ on $I_{q}=\left\{\left(t, s, \lambda, \beta_{0}, \beta, \gamma_{0}\right): 0 \leq t, s<1\right.$, $\left.0 \leq \lambda \leq r_{1}-r_{0}, r_{1}-r_{0} \leq \beta_{0}, \beta, \gamma_{0} \leq \frac{r_{1}-r_{0}}{1-s}\right\}$ by

$$
\begin{aligned}
r_{0} & \geq\left\|y_{0}-x^{0}\right\|, \quad r_{1}>r_{0}+\left\|A\left(y_{0}\right)^{-1}\left(F\left(y_{0}\right)+G\left(y_{0}\right)\right)\right\|, \\
r_{n+1}= & r_{n}+\frac{\int_{0}^{1} \nu\left(t\left(r_{n}-r_{n-1}\right)\right) d t+\omega_{0}\left(r_{n}\right)+\omega\left(r_{n}\right)+b}{1-a-\nu_{0}\left(r_{n}\right)}\left(r_{n}-r_{n-1}\right), \\
f_{n}(s)= & \int_{0}^{1} \nu\left(t s^{n-1}\left(r_{1}-r_{0}\right)\right) d t+\omega_{1}\left(\left(1+s+\cdots+s^{n-1}\right)\left(r_{1}-r_{0}\right)\right)+c, \\
h_{n}(s)= & \int_{0}^{1}\left(\nu\left(t s^{n}\left(r_{1}-r_{0}\right)\right)-\nu\left(t s^{n-1}\left(r_{1}-r_{0}\right)\right) d t\right. \\
& +\omega_{1}\left(\left(1+s+\cdots+s^{n}\right)\left(r_{1}-r_{0}\right)\right) \\
& \quad-\omega_{1}\left(\left(1+s+\cdots+s^{n-1}\right)\left(r_{1}-r_{0}\right)\right),
\end{aligned}
$$

where

$$
\begin{gathered}
c=b-\alpha(1-a) \quad \text { for some } \alpha \in(0,1) \\
\omega_{1}(s)=\omega_{0}(s)+\omega(s)+\alpha \nu_{0}(s) \\
p_{n}(s)=\int_{0}^{1}\left(\nu\left(t s^{n+1}\left(r_{1}-r_{0}\right)\right)\right. \\
\left.+\nu\left(t s^{n-1}\left(r_{1}-r_{0}\right)\right)-2 \nu\left(t s^{n}\left(r_{1}-r_{0}\right)\right)\right) d t \\
+\omega_{1}\left(\left(1+s+\cdots+s^{n+1}\right)\left(r_{1}-r_{0}\right)\right) \\
+\omega_{1}\left(\left(1+s+\cdots+s^{n-1}\right)\left(r_{1}-r_{0}\right)\right) \\
-2 \omega_{1}\left(\left(1+s+\cdots+s^{n}\right)\left(r_{1}-r_{0}\right)\right), \\
q\left(t, s, \lambda, \beta_{0}, \beta, \gamma_{0}\right)=\int_{0}^{1}\left(\nu\left(t \lambda s^{2}\right)+\nu(t \lambda)-2 \nu(t \lambda s)\right) d t \\
\quad+\omega_{1}\left(\beta_{0}+\beta+\gamma_{0}\right)+\omega_{1}(\beta)-2 \omega_{1}\left(\beta_{0}+\beta\right) .
\end{gathered}
$$

Define a function $f_{\infty}$ on $[0,1)$ by

$$
f_{\infty}(s)=\lim _{n \rightarrow \infty} f_{n}(s) .
$$

It then follows from $(2.2)$ and $(2.8)$ that

$$
f_{\infty}(s)=\omega_{1}\left(\frac{r_{1}-r_{0}}{1-s}\right)+c .
$$


It can also be easily seen from $(2.2), 2.3), 2.6)$, and $(2.7)$ that

$$
\begin{gathered}
f_{n+1}(s)=f_{n}(s)+h_{n}(s), \\
h_{n+1}(s)=h_{n}(s)+p_{n}(s), \\
q\left(t, s, s^{n-1}\left(r_{1}-r_{0}\right), s^{n}\left(r_{1}-r_{0}\right),\right. \\
\left.\left(1+s+\cdots+s^{n-1}\right)\left(r_{1}-r_{0}\right), s^{n+1}\left(r_{1}-r_{0}\right)\right)=p_{n}(s) .
\end{gathered}
$$

We need the following result on majorizing sequences for (NTM).

Lemma 2.2. Let constants $a, b$, parameters $r_{0}, r_{1}$, and functions $\nu_{0}, \nu$, $\omega_{0}, \omega$ be as in the Introduction, and let parameters $\alpha, c$ and functions $\omega_{1}$, $f_{n}, h_{n}, p_{n}, q$ be as in Definition 2.1. Assume there exists $\alpha \in(0,1)$ such that

$$
\begin{gathered}
\nu_{0}\left(r_{1}\right)+a<1, \\
\frac{\int_{0}^{1} \nu\left(t\left(r_{1}-r_{0}\right)\right) d t+\omega_{0}\left(r_{1}\right)+\omega\left(r_{1}\right)+b}{1-a-\nu_{0}\left(r_{1}\right)} \leq \alpha, \\
c<0, \\
q\left(t, s, \lambda, \beta_{0}, \beta, \gamma_{0}\right) \geq 0 \quad \text { on } I_{q}, \\
h_{1}(\alpha) \geq 0, \\
f_{\infty}(\alpha) \leq 0 .
\end{gathered}
$$

Then the scalar sequence $\left\{r_{n}\right\}(n \geq 0)$ given by (2.1) is non-decreasing, bounded from above by

$$
r^{\star \star}=\frac{r_{1}-r_{0}}{1-\alpha},
$$

and converges to its least upper bound $r^{\star}$ satisfying $r^{\star} \in\left[0, r^{\star \star}\right]$.

Moreover the following estimates hold for all $n \geq 0$ :

$$
\begin{aligned}
0 \leq r_{n+1}-r_{n} & \leq \alpha\left(r_{n}-r_{n-1}\right) \leq \alpha^{n}\left(r_{1}-r_{0}\right), \\
r^{\star}-r_{n} & \leq \frac{r_{1}-r_{0}}{1-\alpha} \alpha^{n} .
\end{aligned}
$$

Proof. Estimate 2.20 is true if

$$
0 \leq \frac{\int_{0}^{1} \nu\left(t\left(r_{n}-r_{n-1}\right)\right) d t+\omega_{0}\left(r_{n}\right)+\omega\left(r_{n}\right)}{1-a-\nu_{0}\left(r_{n}\right)} \leq \alpha
$$

for all $n \geq 1$.

In view of (2.1), 2.13), 2.14), estimate (2.22) holds true for $n=1$. Also 2.22 implies $0 \leq r_{2}-r_{1} \leq \alpha\left(r_{1}-r_{0}\right)$.

Let us assume that $(2.20)$ and $(2.22)$ hold for all $k \leq n$. Then

$$
r_{n} \leq \frac{1-\alpha^{n}}{1-\alpha}\left(r_{1}-r_{0}\right) .
$$


By the induction hypothesis, and (2.23), estimate 2.22 is true if

$$
\int_{0}^{1} \nu\left(t \alpha^{n-1}\left(r_{1}-r_{0}\right)\right) d t+\omega_{1}\left(\frac{1-\alpha^{n}}{1-\alpha}\left(r_{1}-r_{0}\right)\right)+c \leq 0,
$$

where $c$ and $\omega_{1}$ are given by (2.4) and (2.5), respectively. Estimate 2.24) (for $s=\alpha$ ) motivates us to introduce the function $f_{n}$ given by $(2.2)$, and show, instead of 2.24,

$$
f_{n}(\alpha) \leq 0 \quad(n \geq 1)
$$

By (2.10) 2.12 (for $s=\alpha$ ) and (2.17) we have

$$
f_{n+1}(\alpha) \geq f_{n}(\alpha) \quad(n \geq 1)
$$

In view of (2.8), (2.9), and (2.26), estimate (2.25) holds if $(2.18)$ is true, since

$$
f_{n}(\alpha) \leq f_{\infty}(\alpha) \quad(n \geq 1) .
$$

The induction is completed. It follows that the iteration $\left\{r_{n}\right\}$ is nondecreasing, bounded above by $r^{\star \star}$ (given by (2.19)), and so converges to $r^{\star}$. Finally, estimate (2.21) follows from 2.20 by using standard majorization techniques [4], [17], [18].

That completes the proof of Lemma 2.2 .

The hypotheses $(\mathcal{H})$ and those of Lemma 2.2 will be called $(\mathcal{A})$.

We can now show the main semilocal convergence result for (NTM).

Theorem 2.3. Assume hypotheses $(\mathcal{A})$ hold. Then the sequence $\left\{y_{n}\right\}$ $(n \geq 0)$ generated by $(N T M)$ is well defined, remains in $\bar{U}\left(x^{0}, r^{\star}\right)$ for all $n \geq 0$, and converges to a solution $x^{\star} \in \bar{U}\left(x^{0}, r^{\star}\right)$ of the equation $F(x)+G(x)=0$.

Moreover, the following estimates hold for all $n \geq 0$ :

$$
\begin{aligned}
\left\|y_{n+1}-y_{n}\right\| & \leq r_{n+1}-r_{n}, \\
\left\|y_{n}-x^{\star}\right\| & \leq r^{\star}-r_{n},
\end{aligned}
$$

where the sequence $\left\{r_{n}\right\}(n \geq 0)$ and $r^{\star}$ are given in Lemma 2.2 .

Furthermore, if there exists

$$
R_{0} \in\left[r^{\star}, R\right]
$$

such that

$$
\int_{0}^{1} \nu\left(t\left(R_{0}+r_{0}\right)\right) d t+\omega_{0}\left(r^{\star}\right)+\omega\left(R_{0}\right)+\nu_{0}\left(r^{\star}\right)+a+b \leq 1,
$$

then the solution $x^{\star}$ of equation (1.1) is unique in $U\left(x^{0}, R_{0}\right)$.

Proof. We shall show by induction that

$$
\begin{aligned}
\left\|y_{n}-y_{n-1}\right\| & \leq r_{n}-r_{n-1}, \\
\left\|y_{n}-x^{0}\right\| & \leq r_{n} .
\end{aligned}
$$


Estimates (2.32) and (2.33) hold for $n=1$, by (1.4) and (2.1). Assume they hold for all $n \leq k$. Using $\left(\mathcal{H}_{1}\right)$ and $(2.13)$, we get

$$
\left\|A\left(x_{0}\right)^{-1}\left[A\left(y_{1}\right)-A\left(x^{0}\right)\right]\right\| \leq \nu_{0}\left(\left\|y_{1}-x^{0}\right\|\right)+a \leq \nu_{0}\left(r_{1}\right)+a<1 .
$$

It follows from (2.34) and the Banach lemma on invertible operators [4, 18, that $A\left(y_{1}\right)^{-1}$ exists, and

$$
\left\|A\left(y_{1}\right)^{-1} A\left(x^{0}\right)\right\| \leq\left(1-a-\nu_{0}\left(r_{1}\right)\right)^{-1} .
$$

We also showed in Lemma 2.2 that

$$
\nu_{0}\left(r_{k}\right)+a<1 .
$$

It then follows as in (2.34) with $r_{k}, y_{k}$ replacing $r_{1}, y_{1}$, respectively, that $A\left(y_{k}\right)^{-1}$ exists, and

$$
\left\|A\left(y_{k}\right)^{-1} A\left(x^{0}\right)\right\| \leq\left(1-a-\nu_{0}\left(r_{k}\right)\right)^{-1} .
$$

Using (1.2), $\left.\left.\left(\mathcal{H}_{2}\right),\left(\mathcal{H}_{3}\right),(2.1), 2.32\right), 2.33\right)$, and (2.37), we obtain in turn

$$
\begin{aligned}
\left\|y_{k+1}-y_{k}\right\|= & \left\|A\left(y_{k}\right)^{-1}\left(F\left(y_{k}\right)+G\left(y_{k}\right)\right)\right\| \\
\leq & \left\|A\left(y_{k}\right)^{-1} A\left(x^{0}\right)\right\| \| A\left(x^{0}\right)^{-1}\left(F\left(y_{k}\right)\right. \\
& \left.+G\left(y_{k}\right)-A\left(y_{k-1}\right)\left(y_{k}-y_{k-1}\right)-F\left(y_{k-1}\right)-G\left(y_{k-1}\right)\right) \| \\
\leq & \left(1-a-\nu_{0}\left(r_{k}\right)\right)^{-1}\left(\int_{0}^{1} \| A\left(x^{0}\right)^{-1}\left[F^{\prime}\left(y_{k-1}+\theta\left(y_{k}-y_{k-1}\right)\right)\right.\right. \\
& \left.-A\left(y_{k-1}\right)\right]\|\| y_{k}-y_{k-1} \| d \theta \\
& \left.+\left\|A\left(x^{0}\right)^{-1}\left(G\left(y_{k}\right)-G\left(y_{k-1}\right)\right)\right\|\right) \\
\leq & \left(1-a-\nu_{0}\left(r_{k}\right)\right)^{-1}\left(\int_{0}^{1} \nu\left(t\left\|y_{k}-y_{k-1}\right\|\right) d t\right. \\
& \left.+\omega_{0}\left(\left\|y_{k}-x^{0}\right\|\right)+\omega\left(\left\|y_{k}-x^{0}\right\|\right)+b\right)\left\|y_{k}-y_{k-1}\right\| \\
\leq & \left(1-a-\nu_{0}\left(r_{k}\right)\right)^{-1}\left(\int_{0}^{1} \nu\left(t\left(r_{k}-r_{k-1}\right)\right) d t+\omega_{0}\left(r_{k}\right)\right. \\
& \left.+\omega\left(r_{k}\right)+b\right)\left(r_{k}-r_{k-1}\right)=r_{k+1}-r_{k} .
\end{aligned}
$$

Moreover, we have

$$
\begin{aligned}
\left\|y_{k+1}-x^{0}\right\| & \leq\left\|y_{k+1}-y_{k}\right\|+\left\|y_{k}-x^{0}\right\| \\
& \leq\left(r_{k+1}-r_{k}\right)+r_{k}=r_{k+1} \leq r^{\star} .
\end{aligned}
$$

The induction for $(2.32)$ and $(2.33)$ is completed.

In view of Lemma 2.2 , (2.32), and (2.33), the sequence $\left\{y_{n}\right\}(n \geq 0)$ is Cauchy in the Banach space $\mathcal{X}$, and so it converges to some $x^{\star} \in \bar{U}\left(x^{0}, r^{\star}\right)$ (since $\bar{U}\left(x^{0}, r^{\star}\right)$ is a closed set). 
Estimate $(2.29)$ follows from $(2.28)$ by using standard majorization techniques [4, [17, [18].

Using (2.38), we obtain

$$
\begin{aligned}
& \left\|A\left(x^{0}\right)^{-1}\left(F\left(x_{k}\right)+G\left(x_{k}\right)\right)\right\| \\
& \quad \leq\left(\int_{0}^{1} \nu\left(t\left(r_{k}-r_{k-1}\right)\right) d t+\omega_{0}\left(r_{k}\right)+\omega\left(r_{k}\right)+b\right)\left(r_{k}-r_{k-1}\right) .
\end{aligned}
$$

By letting $k \rightarrow \infty$ in 2.40 , we obtain $F\left(x^{\star}\right)+G\left(x^{\star}\right)=0$. Finally to show that $x^{\star}$ is the unique solution of equation 1.1) in $\bar{U}\left(x^{0}, R_{0}\right)$, let $y^{\star} \in$ $\bar{U}\left(x^{0}, R_{0}\right)$, with $F\left(y^{\star}\right)+G\left(y^{\star}\right)=0$.

Using the approximation

$$
\begin{aligned}
& y^{\star}-y_{k+1} \\
& \quad=y^{\star}-y_{k}+A\left(y_{k}\right)^{-1}\left(F\left(y_{k}\right)+G\left(y_{k}\right)\right)-A\left(y_{k}\right)^{-1}\left(F\left(y^{\star}\right)+G\left(y^{\star}\right)\right),
\end{aligned}
$$

as in (2.38), we obtain in turn

$$
\begin{aligned}
\leq & \left(1-a-\nu_{0}\left(r_{k}\right)\right)^{-1}\left(\int_{0}^{1} \| A\left(x^{0}\right)^{-1}\left[F^{\prime}\left(y_{k}+\theta\left(y^{\star}-y_{k}\right)\right)\right.\right. \\
& \left.\left.-A\left(y_{k}\right)\right]\|\| y^{\star}-y_{k}\|d \theta+\| A\left(x^{0}\right)^{-1}\left(G\left(y^{\star}\right)-G\left(y_{k}\right)\right) \|\right) \\
\leq & \left(1-a-\nu_{0}\left(r_{k}\right)\right)^{-1}\left(\int_{0}^{1} \nu\left(t\left\|y^{\star}-y_{k}\right\|\right) d t+\omega_{0}\left(\left\|y_{k}-x^{0}\right\|\right)\right. \\
& \left.+\omega\left(\left\|y_{k}-x^{0}\right\|\right)+b\right)\left\|y^{\star}-y_{k}\right\| \\
< & \left(1-a-\nu_{0}\left(r^{\star}\right)\right)^{-1}\left(\int_{0}^{1} \nu\left(t\left(R_{0}+r_{0}\right)\right) d t+\omega_{0}\left(r^{\star}\right)+\omega\left(R_{0}\right)+b\right)\left\|y_{k}-y^{\star}\right\| \\
\leq & \left\|y_{k}-y^{\star}\right\| \quad(\text { by } 2.31) .
\end{aligned}
$$

It follows from (2.42) that $\lim _{k \rightarrow \infty} y_{k}=y^{\star}$. But we showed $\lim _{k \rightarrow \infty} y_{k}=x^{\star}$. Hence, $x^{\star}=y^{\star}$.

That completes the proof of Theorem 2.3 .

\section{Special cases and applications}

Application 3.1 (Newton's method). Let $A(x)=F^{\prime}(x)$ and $G(x)=0$ $(x \in \mathcal{D})$. Then, in the case of the $(\mathcal{C})$ conditions, we have

$$
\begin{gathered}
y_{0}=x^{0}=x_{0}, \quad s_{0}=0, \quad s_{1}=\eta, \quad a=b=0, \\
\overline{\nu_{0}}(r)=L_{0} r, \quad \bar{\nu}(r)=L r, \quad \omega(r)=0,
\end{gathered}
$$




$$
\begin{gathered}
\chi(r)=\phi(r)=\frac{L}{2} r^{2}-r+\eta, \quad \chi^{\star}=\frac{2 L \eta-1}{2 L}, \\
s^{\star}=\frac{1}{L}, \quad t^{\star}=\frac{1-\sqrt{1-2 L \eta}}{L}, \quad R \in\left[t^{\star}, s^{\star}\right], \\
u(r)=\frac{1}{2 L}(L r-1)^{2}, \quad p(r)=1-L_{0}, r, \\
h_{C Y}=L \eta \leq 1 / 2,
\end{gathered}
$$

and

$$
\begin{aligned}
& s_{0}=0, \quad s_{1}=\eta, \\
& s_{n+1}=s_{n}+\frac{\left(L s_{n}-1\right)^{2}}{2 L\left(1-L_{0} s_{n}\right)}=s_{n}+\frac{L\left(s_{n}-s_{n-1}\right)^{2}}{2\left(1-L_{0} s_{n}\right)} \quad(n \geq 1),
\end{aligned}
$$

Moreover, in the case of the $(\mathcal{A})$ conditions, we have:

$$
\begin{gathered}
y_{0}=x^{0}=x_{0}, \quad r_{0}=0, \quad r_{1}=\eta, \quad a=b=0, \\
\nu_{0}(r)=L_{0} r, \quad \nu(r)=L r, \quad \omega(r)=\omega_{0}(r)=0, \\
\alpha=\frac{2 L}{L+\sqrt{L^{2}+8 L_{0} L}}, \\
h_{A H}=\bar{L} \eta \leq 1 / 2,
\end{gathered}
$$

where

$$
\bar{L}=\frac{1}{8}\left(L+4 L_{0}+\sqrt{L^{2}+8 L_{0} L}\right)
$$

and

$$
\begin{aligned}
& s_{0}=0, \quad s_{1}=\eta \\
& s_{n+1}=s_{n}+\frac{L\left(s_{n}-s_{n-1}\right)^{2}}{2\left(1-L_{0} s_{n}\right)} \quad(n \geq 1) .
\end{aligned}
$$

Note that

$$
L_{0} \leq L
$$

in general, and $L / L_{0}$ can be arbitrarily large [4]-[7].

Let us now compare the results. It follows from (3.1), (3.3), and 3.5 that

$$
h_{C Y} \leq 1 / 2 \Rightarrow h_{A H} \leq 1 / 2,
$$

but not necessarily vice versa unless $L_{0}=L$.

Hence the convergence domains approach in [11] does not necessarily produce the weakest sufficient convergence conditions, even in the simplest possible case of a Newton-like method which is Newton's method (1.8). The recurrent functions approach produces sufficient convergence 3.3 that can always replace $(3.1)$. 
Hence, the applicability of Newton's method has been extended, under the same hypotheses and computational cost as in [11, [18. Note that the results in [15], 22], 23] are also improved in at least the Newton's method case, since their conditions also lead to (3.1) instead of (3.3).

In the Newton's method case, although the majorizing sequences $\left\{s_{n}\right\}$ and $\left\{r_{n}\right\}$ coincide, the convergence domains approach fails to take advantage of the relationship between $L_{0}$ and $L$, since $L_{0}$ does not appear in (3.1). The same is happening in the general case, since the function $\chi$ does not depend on $\overline{\nu_{0}}$. However, our approach depends on $\nu_{0}$ for the derivation of the sufficient convergence conditions. Under our method the ratio " $\alpha$ " of convergence for $\left\{s_{n}\right\}$ is known, but this is not true for the iteration $\left\{r_{n}\right\}$.

Next, we provide three examples where $L_{0}<L$. Moreover, in the first example, (3.3) is satisfied but (3.1) is not.

EXAMPLE 3.2.

Case 1: $A(x)=F^{\prime}(x)$ and $G(x)=0(x \in \mathcal{D})$. Let $\mathcal{X}=\mathcal{Y}=\mathbb{R}^{2}$, equipped with the max-norm, $x^{0}=(1,1)^{T}$, and

$$
\mathcal{D}=U_{0}=\left\{x:\left|x-x^{0}\right| \leq 1-\delta\right\}, \quad \delta \in[0,1 / 2) .
$$

Define a function $F$ on $U_{0}$ by

$$
F(x)=\left(\xi_{1}^{3}-\delta, \xi_{2}^{3}-\delta\right), \quad x=\left(\xi_{1}, \xi_{2}\right)^{T} .
$$

The Fréchet derivative of $F$ is given by

$$
F^{\prime}(x)=\left[\begin{array}{cc}
3 \xi_{1}^{2} & 0 \\
0 & 3 \xi_{2}^{2}
\end{array}\right]
$$

Using the hypotheses of Theorem 2.3 , we get

$$
\eta=\frac{1}{3}(1-\delta), \quad L=3-\delta, \quad K=2(2-\delta) .
$$

The Newton-Kantorovich condition (3.1) is violated, since

$$
\frac{4}{3}(1-\delta)(2-\delta)>1 \quad \text { for all } \delta \in[0,1 / 2) .
$$

Hence, there is no guarantee that Newton's method $(1.2)$ converges to $x^{\star}=$ $(\sqrt[3]{\delta}, \sqrt[3]{\delta})^{T}$, starting at $x^{0}$

However, our condition (3.3) is true for all $\delta \in I=[.450339002,1 / 2)$. Hence, the conclusions of our Theorem 2.3 can be applied to solve equation (3.7) for all $\delta \in I$.

CASE 2: $A(x)=F^{\prime}(x)$ and $G(x)=\left(\epsilon\left|\xi_{1}-1\right|, \epsilon\left|\xi_{2}-1\right|\right)(x \in \mathcal{D})$. Let us choose $\epsilon=.1, \delta=.49, y_{0}=x^{0}$. Using the $(\mathcal{C})$ conditions, we have

$$
r_{0}=0, \quad \overline{\nu_{0}}(r)=\bar{\nu}(r)=\ell r, \quad \omega(r)=|\epsilon|, \quad r \geq 0, \quad a=b=0,
$$


and

$$
\chi(r)=\frac{\ell}{2} r^{2}-(1-|\epsilon|) r+\eta .
$$

However, the discriminant of the function $\chi$ is negative, since

$$
\frac{4}{3}(1-\delta)(2-\delta)>(1-|\epsilon|)^{2} \quad \text { for all } \delta \in[0,1 / 2) .
$$

Hence, the $(\mathcal{C})$ conditions do not hold. That is, there is no guarantee that the iteration 1.2 converges to a solution $x^{\star}$ of equation (1.1).

We shall now check condition $(\mathcal{A})$. We have

$$
\begin{gathered}
\nu_{0}(r)=\ell_{0} r, \quad \ell_{0}=2.51, \quad \omega_{0}(r)=0, \\
\nu(r)=\ell r, \quad \ell=3.02, \quad \omega(r)=\ell_{1}=|\epsilon|=.1 .
\end{gathered}
$$

Choose $r_{1}=.118$. Then 2.13 -2.18 hold, since by Maple we have

$$
.118<\frac{1}{\ell_{0}} \simeq .398406374, \quad \frac{\int_{0}^{1} \ell t r_{1} d t+.1}{1-\ell_{0} r_{1}} \leq \alpha,
$$

or

$$
\alpha \geq .395243102
$$

so we choose

$$
\alpha=.40353, \quad c=-\alpha<0, \quad \omega_{1}(r)=.1+\alpha \ell_{0} r,
$$

Then

$$
\begin{aligned}
f_{\infty}(\alpha) & =.1+\frac{\alpha \ell_{0} r_{1}}{1-\alpha}-\alpha=-.1031552697<0 \\
h_{1}(r) & =\left(\left(\frac{\ell}{2}+\alpha \ell_{0}\right) r+\alpha \ell_{0}-\frac{\ell}{2}\right) r_{1}
\end{aligned}
$$

SO

$$
h_{1}(\alpha)=.06146739381 \geq 0, \quad R=r^{\star \star}=.1978305699,
$$

and

$$
\bar{U}\left(x^{0}, R\right) \subseteq U\left(x^{0}, 1-\delta\right)=U\left(x^{0}, .51\right) .
$$

Hence, all hypotheses of Theorem 2.3 are satisfied. That is, the iteration (NTM) starting from $x^{0}$ converges to $x^{\star}=(.788373516, .788373516)^{T}$.

ExAmple 3.3. Let $\mathcal{X}=\mathcal{Y}=\mathcal{C}[0,1]$ be the space of real-valued continuous functions defined on the interval $[0,1]$ with the norm

$$
\|x\|=\max _{0 \leq s \leq 1}|x(s)| \text {. }
$$

Let $\theta \in[0,1]$ be a given parameter. Consider the "cubic" integral equation

$$
u(s)=u^{3}(s)+\lambda u(s) \int_{0}^{1} q(s, t) u(t) d t+y(s)-\theta .
$$


Here the kernel $q(s, t)$ is a continuous function of two variables defined on $[0,1] \times[0,1] ; \lambda$ is a real number called the "albedo" for scattering; $y(s)$ is a given continuous function defined on $[0,1]$; and $x(s)$ is the unknown function sought in $\mathcal{C}[0,1]$. Equations of the form (3.8) arise in the kinetic theory of gasses [4], [10]. For simplicity, we choose $u_{0}(s)=y(s)=1$, and $q(s, t)=s /(s+t)$ for all $s \in[0,1]$ and $t \in[0,1]$ with $s+t \neq 0$. If we let $\mathcal{D}=U\left(u_{0}, 1-\theta\right)$, and define the operator $F$ on $\mathcal{D}$ by

$$
F(x)(s)=x^{3}(s)-x(s)+\lambda x(s) \int_{0}^{1} q(s, t) x(t) d t+y(s)-\theta
$$

for all $s \in[0,1]$, then every zero of $F$ satisfies equation 3.8$)$. We have the estimate

$$
\max _{0 \leq s \leq 1}\left|\int_{0}^{1} \frac{s}{s+t} d t\right|=\ln 2 .
$$

Therefore, if we set $\xi=\left\|F^{\prime}\left(u_{0}\right)^{-1}\right\|$, then it follows from the hypotheses of Theorem 2.3 that

$$
\begin{gathered}
\eta=\xi(|\lambda| \ln 2+1-\theta), \\
L=2 \xi(|\lambda| \ln 2+3(2-\theta)) \quad \text { and } \quad L_{0}=\xi(2|\lambda| \ln 2+3(3-\theta)) .
\end{gathered}
$$

It follows from Theorem 2.3 that if condition (3.3) holds, then problem (3.8) has a unique solution near $u_{0}$. This assumption is weaker than the one given before using the Newton-Kantorovich hypothesis (3.1).

Note also that $L_{0}<L$ for all $\theta \in[0,1]$.

EXAMPLE 3.4. Consider the following nonlinear boundary value problem [4]:

$$
\left\{\begin{array}{l}
u^{\prime \prime}=-u^{3}-\rho u^{2} \\
u(0)=0, \quad u(1)=1 .
\end{array}\right.
$$

It is well known that this problem can be formulated as the integral equation

$$
u(s)=s+\int_{0}^{1} Q(s, t)\left(u^{3}(t)+\rho u^{2}(t)\right) d t
$$

where $Q$ is the Green function

$$
Q(s, t)= \begin{cases}t(1-s), & t \leq s \\ s(1-t), & s<t\end{cases}
$$

We observe that

$$
\max _{0 \leq s \leq 1} \int_{0}^{1}|Q(s, t)|=\frac{1}{8}
$$


Let $\mathcal{X}=\mathcal{Y}=\mathcal{C}[0,1]$ with the norm

$$
\|x\|=\max _{0 \leq s \leq 1}|x(s)| .
$$

Then problem (3.10) is in the form (1.1), where $F: \mathcal{D} \rightarrow \mathcal{Y}$ is defined as

$$
[F(x)](s)=x(s)-s-\int_{0}^{1} Q(s, t)\left(x^{3}(t)+\rho x^{2}(t)\right) d t,
$$

and

$$
G(x)(s)=0 .
$$

It is easy to verify that the Fréchet derivative of $F$ is

$$
\left[F^{\prime}(x) v\right](s)=v(s)-\int_{0}^{1} Q(s, t)\left(3 x^{2}(t)+2 \rho x(t)\right) v(t) d t .
$$

If we set $u_{0}(s)=s$ and $\mathcal{D}=U\left(u_{0}, R\right)$, then since $\left\|u_{0}\right\|=1$, it is easy to verify that $U\left(u_{0}, R\right) \subset U(0, R+1)$. It follows that if $2 \rho<5$, then

$$
\begin{aligned}
\left\|I-F^{\prime}\left(u_{0}\right)\right\| & \leq \frac{3\left\|u_{0}\right\|^{2}+2 \rho\left\|u_{0}\right\|}{8}=\frac{3+2 \rho}{8}, \\
\left\|F^{\prime}\left(u_{0}\right)^{-1}\right\| & \leq \frac{1}{1-\frac{3+2 \rho}{8}}=\frac{8}{5-2 \rho}, \\
\left\|F\left(u_{0}\right)\right\| & \leq \frac{\left\|u_{0}\right\|^{3}+\rho\left\|u_{0}\right\|^{2}}{8}=\frac{1+\rho}{8}, \\
\left\|F\left(u_{0}\right)^{-1} F\left(u_{0}\right)\right\| & \leq \frac{1+\rho}{5-2 \rho} .
\end{aligned}
$$

On the other hand, for $x, y \in \mathcal{D}$, we have

$$
\left[\left(F^{\prime}(x)-F^{\prime}(y)\right) v\right](s)=-\int_{0}^{1} Q(s, t)\left(3 x^{2}(t)-3 y^{2}(t)+2 \rho(x(t)-y(t))\right) v(t) d t .
$$

Consequently,

$$
\begin{aligned}
\left\|F^{\prime}(x)-F^{\prime}(y)\right\| & \leq \frac{\|x-y\|(2 \rho+3(\|x\|+\|y\|))}{8} \\
& \leq \frac{\|x-y\|\left(2 \rho+6 R+6\left\|u_{0}\right\|\right)}{8} \\
& =\frac{\rho+6 R+3}{4}\|x-y\|, \\
\left\|F^{\prime}(x)-F^{\prime}\left(u_{0}\right)\right\| & \leq \frac{\left\|x-u_{0}\right\|\left(2 \rho+3\left(\|x\|+\left\|u_{0}\right\|\right)\right)}{8} \\
& \leq \frac{\left\|x-u_{0}\right\|\left(2 \rho+3 R+6\left\|u_{0}\right\|\right)}{8} \\
& =\frac{2 \rho+3 R+6}{8}\left\|x-u_{0}\right\| .
\end{aligned}
$$


Therefore, the conditions of Theorem 2.3 hold with

$$
\eta=\frac{1+\rho}{5-2 \rho}, \quad L=\frac{\rho+6 R+3}{4}, \quad L_{0}=\frac{2 \rho+3 R+6}{8} .
$$

Note also that $L_{0}<L$.

Finally, we provide an example to show that method 1.6 is faster than method 1.4 .

ExAmple $3.5([9])$. Let $\mathcal{X}=\mathcal{Y}=\left(\mathbb{R}^{2},\|\cdot\|_{\infty}\right)$. Consider the system

$$
\begin{aligned}
3 x^{2} y+y^{2}-1+|x-1| & =0, \\
x^{4}+x y^{3}-1+|y| & =0 .
\end{aligned}
$$

For $v=\left(v_{1}, v_{2}\right)$ set

$$
\|v\|_{\infty}=\left\|\left(v_{1}, v_{2}\right)\right\|_{\infty}=\max \left\{\left|v_{1}\right|,\left|v_{2}\right|\right\}, \quad F=\left(F_{1}, F_{2}\right), \quad G=\left(G_{1}, G_{2}\right),
$$

where

$$
\begin{array}{llrl}
F_{1}(v) & =3 v_{1}^{2} v_{2}+v_{2}^{2}-1, & & F_{2}(v)=v_{1}^{4}+v_{1} v_{2}^{3}-1 \\
G_{1}(v)=\left|v_{1}-1\right|, & G_{2}(v)=\left|v_{2}\right| .
\end{array}
$$

The divided differences of order one $[x, y ; F],[x, y ; G] \in \mathcal{M}_{2 \times 2}(\mathbb{R})$ are for $w=\left(w_{1}, w_{2}\right)$,

$$
[v, w, F]_{i, 1}=\frac{F_{i}\left(w_{1}, w_{2}\right)-F_{i}\left(v_{1}, w_{2}\right)}{w_{1}-v_{1}}, \quad[v, w, F]_{i, 2}=\frac{F_{i}\left(v_{1}, w_{2}\right)-F_{i}\left(v_{1}, v_{2}\right)}{w_{2}-v_{2}}
$$

provided that $w_{1} \neq v_{1}$ and $w_{2} \neq v_{2}$. If $w_{1}=v_{1}$ or $w_{2}=v_{2}$, replace $[x, y ; F]$ by $F^{\prime}$. Similarly we define

$$
[v, w ; G]_{i, 1}=\frac{G_{i}\left(w_{1}, w_{2}\right)-G_{i}\left(v_{1}, w_{2}\right)}{w_{1}-v_{1}}, \quad[v, w ; G]_{i, 2}=\frac{G_{i}\left(v_{1}, w_{2}\right)-G_{i}\left(v_{1}, v_{2}\right)}{w_{2}-v_{2}}
$$

for $w_{1} \neq v_{1}$ and $w_{2} \neq v_{2}$. If $w_{1}=v_{1}$ or $w_{2}=v_{2}$, replace $[x, y ; G]$ by the zero $2 \times 2$ matrix in $\mathcal{M}_{2 \times 2}(\mathbb{R})$.

We consider three interesting choices for the operator $A$ :

$$
\begin{aligned}
A(v, w) & =F(v)+G(v)+F^{\prime}(v)(w-v), \\
A(u, v, w) & =F(v)+G(v)+([u, v ; F]+[u, v ; G])(w-v), \\
A(u, v, w) & =F(v)+G(v)+\left(F^{\prime}(v)+[u, v ; G]\right)(w-v) .
\end{aligned}
$$

Using the method $(1.4)$, based on the operator 3.12 for $y_{0}=(1,0)^{T}$, and the methods based on the operators $(3.13)$ and $(3.14)$ (the latter is $(1.6)$ ) for $y_{-1}=(5,5)^{T}, y_{0}=(1,0)^{T}$, we obtain the following three tables respectively: 


\begin{tabular}{llll}
\hline$n$ & $y_{n}^{(1)}$ & $y_{n}^{(2)}$ & $\left\|y_{n}-y_{n-1}\right\|$ \\
\hline 0 & 1 & 0 & \\
1 & 1 & 0.333333333333333 & $3.333 \mathrm{E}-1$ \\
2 & 0.906550218340611 & 0.354002911208151 & $9.344 \mathrm{E}-2$ \\
3 & 0.885328400663412 & 0.338027276361322 & $2.122 \mathrm{E}-2$ \\
4 & 0.891329556832800 & 0.326613976593566 & $1.141 \mathrm{E}-2$ \\
5 & 0.895238815463844 & 0.326406852843625 & $3.909 \mathrm{E}-3$ \\
6 & 0.895154671372635 & 0.327730334045043 & $1.323 \mathrm{E}-3$ \\
7 & 0.894673743471137 & 0.327979154372032 & $4.809 \mathrm{E}-4$ \\
8 & 0.894598908977448 & 0.327865059348755 & $1.140 \mathrm{E}-4$ \\
9 & 0.894643228355865 & 0.327815039208286 & $5.002 \mathrm{E}-5$ \\
10 & 0.894659993615645 & 0.327819889264891 & $1.676 \mathrm{E}-5$ \\
11 & 0.894657640195329 & 0.327826728208560 & $6.838 \mathrm{E}-6$ \\
12 & 0.894655219565091 & 0.327827351826856 & $2.420 \mathrm{E}-6$ \\
13 & 0.894655074977661 & 0.327826643198819 & $7.086 \mathrm{E}-7$ \\
$\ldots$ & & & \\
39 & 0.894655373334687 & 0.327826521746298 & $5.149 \mathrm{E}-19$ \\
\hline
\end{tabular}

\begin{tabular}{rlll}
\hline$n$ & $y_{n}^{(1)}$ & $y_{n}^{(2)}$ & $\left\|y_{n}-y_{n-1}\right\|$ \\
\hline-1 & 5 & 5 & \\
0 & 1 & 0 & $5.000 \mathrm{E}+00$ \\
1 & 0.989800874210782 & 0.012627489072365 & $1.262 \mathrm{E}-02$ \\
2 & 0.921814765493287 & 0.307939916152262 & $2.953 \mathrm{E}-01$ \\
3 & 0.900073765669214 & 0.325927010697792 & $2.174 \mathrm{E}-02$ \\
4 & 0.894939851625105 & 0.327725437396226 & $5.133 \mathrm{E}-03$ \\
5 & 0.894658420586013 & 0.327825363500783 & $2.814 \mathrm{E}-04$ \\
6 & 0.894655375077418 & 0.327826521051833 & $3.045 \mathrm{E}-04$ \\
7 & 0.894655373334698 & 0.327826521746293 & $1.742 \mathrm{E}-09$ \\
8 & 0.894655373334687 & 0.327826521746298 & $1.076 \mathrm{E}-14$ \\
9 & 0.894655373334687 & 0.327826521746298 & $5.421 \mathrm{E}-20$ \\
\hline
\end{tabular}

\begin{tabular}{rlll}
\hline$n$ & $y_{n}^{(1)}$ & $y_{n}^{(2)}$ & $\left\|y_{n}-y_{n-1}\right\|$ \\
\hline-1 & 5 & 5 & \\
0 & 1 & 0 & 5 \\
1 & 0.909090909090909 & 0.363636363636364 & $3.636 \mathrm{E}-01$ \\
2 & 0.894886945874111 & 0.329098638203090 & $3.453 \mathrm{E}-02$ \\
3 & 0.894655531991499 & 0.327827544745569 & $1.271 \mathrm{E}-03$ \\
4 & 0.894655373334793 & 0.327826521746906 & $1.022 \mathrm{E}-06$ \\
5 & 0.894655373334687 & 0.327826521746298 & $6.089 \mathrm{E}-13$ \\
6 & 0.894655373334687 & 0.327826521746298 & $2.710 \mathrm{E}-20$ \\
\hline
\end{tabular}


We have not verified the hypotheses of Theorem 2.3 for the above starting points. However, it is clear that the hypotheses of Theorem 2.3 are satisfied for all three methods for starting points closer to the solution

$$
x^{\star}=(.894655373334687, .327826521746298)^{T}
$$

chosen from the lists of the tables displayed above.

Hence method (1.6) for choice (3.14) converges faster than (1.4) suggested in Chen and Yamamoto [11, Zabrejko and Nguen [26] in this case and the method of chord [4, 6], [27].

Conclusion. Using our new idea of recurrent functions, and ZabrejkoZinčenko-type conditions, we provided a semilocal convergence analysis for (NTM) in order to approximate a locally unique solution of an equation in a Banach space. Our analysis has the following advantages over the work in [11]: weaker sufficient convergence conditions and larger convergence domain. Note that in the case of Newton's method, these advantages are obtained under the same computational cost, since in practice the computation of the Lipschitz constant $L$ requires the computation of $L_{0}$. Numerical examples further validating the results are also provided in this study.

\section{References}

[1] I. K. Argyros, The Theory and Application of Abstract Polynomial Equations, St.Lucie/CRC/Lewis Publ., Boca Raton, FL, 1998.

[2] —, On the Newton-Kantorovich hypothesis for solving equations, J. Comput. Appl. Math. 169 (2004), 315-332.

[3] - A unifying local-semilocal convergence analysis and applications for two-point Newton-like methods in Banach space, J. Math. Anal. Appl. 298 (2004), 374-397.

[4] - Convergence and Applications of Newton-type Iterations, Springer, New York, 2008.

[5] - A semilocal convergence analysis for directional Newton methods, Math. Comp. 80 (2011), 327-343.

[6] I. K. Argyros and S. Hilout, Efficient Methods for Solving Equations and Variational Inequalities, Polimetrica, Milano, 2009.

[7] —, - Aspects of the Computational Theory for Certain Iterative Methods, Polimetrica, Milano, 2009.

[8] - - - Enclosing roots of polynomial equations and their applications to iterative processes, Surveys Math. Appl. 4 (2009), 119-132.

[9] E. Cătinaş, On some iterative methods for solving nonlinear equations, Rev. Anal. Numér. Théorie Approx. 23 (1994), 47-53.

[10] S. Chandrasekhar, Radiative Transfer, Dover Publ., New York, 1960.

[11] X. Chen and T. Yamamoto, Convergence domains of certain iterative methods for solving nonlinear equations, Numer. Funct. Anal. Optim. 10 (1989), 37-48.

[12] J. E. Dennis, Toward a unified convergence theory for Newton-like methods, in: Nonlinear Functional Analysis and Applications, L. B. Rall (ed.), Academic Press, New York, 1971, 425-472. 
[13] P. Deuflhard, Newton Methods for Nonlinear Problems. Affine Invariance and Adaptive Algorithms, Springer Ser. Comput. Math. 35, Springer, Berlin, 2004.

[14] P. Deuflhard and G. Heindl, Affine invariant convergence theorems for Newton's method and extensions to related methods, SIAM J. Numer. Anal. 16 (1979), 1-10.

[15] J. A. Ezquerro and M. A. Hernández, Generalized differentiability conditions for Newton's method, IMA J. Numer. Anal. 22 (2002), 187-205.

[16] Z. Huang, A note on the Kantorovich theorem for Newton iteration, J. Comput. Appl. Math. 47 (1993), 211-217.

[17] L. V. Kantorovich, On Newton's method for functional equations, Dokl. Akad. Nauk SSSR 59 (1948), 1237-1240 (in Russian).

[18] L. V. Kantorovich and G. P. Akilov, Functional Analysis, Pergamon Press, Oxford, 1982.

[19] F. A. Potra, On the convergence of a class of Newton-like methods, in: Iterative Solution of Nonlinear Systems of Equations (Oberwolfach, 1982), Lecture Notes in Math. 953, Springer, Berlin, 1982, 125-137.

[20] —, On an iterative algorithm of order $1.839 \ldots$ for solving nonlinear operator equations, Numer. Funct. Anal. Optim. 7 (1984/85), 75-106.

[21] —, Sharp error bounds for a class of Newton-like methods, Libertas Math. 5 (1985), 71-84.

[22] P. D. Proinov, General local convergence theory for a class of iterative processes and its applications to Newton's method, J. Complexity 25 (2009), 38-62.

[23] - New general convergence theory for iterative processes and its applications to Newton-Kantorovich type theorems, ibid. 26 (2010), 3-42.

[24] W. C. Rheinboldt, A unified convergence theory for a class of iterative processes, SIAM J. Numer. Anal. 5 (1968), 42-63.

[25] T. Yamamoto, A convergence theorem for Newton-like methods in Banach spaces, Numer. Math. 51 (1987), 545-557.

[26] P. P. Zabrejko and D. F. Nguen, The majorant method in the theory of NewtonKantorovich approximations and the Pták error estimates, Numer. Funct. Anal. Optim. 9 (1987), 671-684.

[27] A. I. Zinčenko, Some approximate methods of solving equations with non-differentiable operators, Dopov. Akad. Nauk Ukraïn. RSR 1963, 156-161 (in Ukrainian).

Ioannis K. Argyros

Department of Mathematical Sciences

Cameron University

Lawton, OK 73505, U.S.A.

E-mail: iargyros@cameron.edu
Saïd Hilout Laboratoire de Mathématiques et Applications Poitiers University Bd. Pierre et Marie Curie, Téléport 2, B.P. 30179 86962 Futuroscope Chasseneuil Cedex, France E-mail: said.hilout@math.univ-poitiers.fr

Received on 26.1.2010;

revised version on 23.6.2010 
